OPEN ACCESS

Edited by:

Baokai Cui,

Beijing Forestry University, China

Reviewed by:

Dapeng Bao,

Institute of Edible Fungi (SAAS), China

Gerardo Díaz-Godínez,

Autonomous University of Tlaxcala,

Mexico

*Correspondence:

Yu Hua Gong

gongyuhua@mail.hzau.edu.cn

†These authors have contributed equally to this work

Specialty section This article was submitted to Fungi and Their Interactions,

a section of the journal

Frontiers in Microbiology

Received: 24 December 2019

Accepted: 26 March 2020

Published: 17 April 2020

Citation:

Wang G, Luo Y, Wang C, Zhou Y,

Mou C, Kang H, Xiao Y, Bian Y and

Gong YH (2020) Hsp40 Protein

LeDnaJ07 Enhances

the Thermotolerance of Lentinula

edodes and Regulates IAA

Biosynthesis by Interacting LetrpE.

Front. Microbiol. 11:707.

doi: 10.3389/fmich.2020.00707

\section{Hsp40 Protein LeDnaJ07 Enhances the Thermotolerance of Lentinula edodes and Regulates IAA Biosynthesis by Interacting LetrpE}

\author{
Gangzheng Wang ${ }^{1,2 t}$, Yi Luo ${ }^{1 \dagger}$, Chen Wang ${ }^{1}$, Yan Zhou ${ }^{1}$, Chunye Mou ${ }^{1}$, Heng Kang ${ }^{1}$, \\ Yang Xiao ${ }^{1}$, Yinbing Bian ${ }^{1}$ and Yu Hua Gong ${ }^{1 *}$
}

'Institute of Applied Mycology, College of Plant Science and Technology, Huazhong Agricultural University, Wuhan, China, 2 State Key Laboratory of Applied Microbiology Southern China, Guangdong Provincial Key Laboratory of Microbial Culture Collection and Application, Guangdong Open Laboratory of Applied Microbiology, Guangdong Institute of Microbiology, Guangzhou, China

Our previous study found that LeDnaJ07 RNAi decreased Lentinula edodes resistance to heat stress and Trichoderma atroviride infection. In this study, the structure and function of the LeDnaJ07 gene was analyzed by gene cloning and overexpression in L. edodes stress-sensitive strain YS55 via the Agrobacterium-mediated transformation method. Transformants were confirmed by qRT-PCR, fluorescence observation and Southern blotting. Overexpression of LeDnaJ07 in YS55 not only enhanced L. edodes mycelial resistance to heat stress but also facilitated mycelial growth. In the presence of heat stress, the intracellular IAA content showed a significant increase in the two LeDnaJ07 overexpression strains but only a slight change in the YS55 wild type strain. Moreover, the interaction between LeDnaJ07 and LetrpE was demonstrated via Y2H and BiFC assays. These results suggested that LeDnaJ07 may be involved in regulating IAA biosynthesis and the resistance of $L$. edodes to heat stresses via interacting with LetrpE.

Keywords: DnaJ protein, Lentinula edodes, thermotolerance, IAA, yeast two-hybrid, BiFC, LetrpE

\section{INTRODUCTION}

Heat stress is a major constraint in mushroom production. High temperature not only inhibits mycelial growth and fruiting body formation, but also enhances Trichoderma sp. infection and cell membrane fluidity in edible fungi (Lu et al., 2014; Liu et al., 2017; Qiu et al., 2017). Previous studies have reported that Para-aminobenzoic acid (PABA) synthase and nitric oxide reduced ROS (reactive oxygen species) accumulation to alleviate oxidative damage induced by heat stress (Kong et al., 2012a; Lu et al., 2014); catalase, trehalose, HSP and calcium-calmodulin play an important role in regulating the response of the edible fungi to heat stress (Kong et al., 2012b; Deng et al., 2014; Wang et al., 2017; Liu et al., 2018).

Lentinula edodes, one mushroom used for decoctions and essences and alternative medicine, is widely cultivated in the world and ranks second in worldwide production next to Agaricus bisporus (Chang, 2005; Finimundy et al., 2014). During mycelial culture of L. edodes in rotted logs or bags, six Trichoderma species have been isolated and identified in previous studies, and these studies 
reported that high temperatures caused large losses in L. edodes yield (Cao et al., 2015; Wang G.Z. et al., 2016). Our previous studies found that LeDnaJ07 (named as LeDnaJ in our previous reports) and auxin participated in the response to heat stress in L. edodes (Wang et al., 2018a,b; Zhou et al., 2018).

Heat shock protein 40 (termed DnaJ or J protein), an important member of the heat shock protein family, plays a critical role in modulating the growth and development processes of organism as well as their resistance to abiotic and biotic stresses (Caplan et al., 1992; Suetsugu et al., 2005; Shen and Yu, 2011; Zhou et al., 2012). In plants, the function of DnaJ protein is well-known. DnaJ proteins regulate the resistance of plants to abiotic stresses (heat, cold, salt, and drought) via inducing cell elongation and stabilizing the structure of the photosynthetic system II (Bekhochir et al., 2013; Kong et al., 2014b; Wang et al., 2019). In addition, overexpression of DnaJ protein enhanced the resistance of tobacco to Phytophthora parasitica pv nicotianae and Sclerotinia sclerotiorum via regulating the activity of ascorbic acid peroxidase (APX), Mn-SOD (superoxide) and HSP70 (Rampuria et al., 2018). Moreover, the knockdown of OsDjA6 enhanced the resistance of Oryza sativa to Magnaporthe oryzae (Zhong et al., 2018). For Candida albicans, Ydj1 loss resulted in the failure of morphogenetic switch from yeast to filamentous form (Xie et al., 2017). However, the function of DnaJ protein in macro basidiomycete fungi is less well-known.

Based on our previous studies, the transcript and protein levels of LeDnaJ07 were dramatically upregulated in the heatresistant strain S606 after heat stress. LeDnaJ07 overexpression in L. edodes strain S606 enhanced the thermotolerance via regulating IAA biosynthesis, while LeDnaJ07 RNAi in L. edodes strain $\$ 606$ played a negative role in regulating mycelial growth, the resistance to heat stress and $T$. atroviride and IAA biosynthesis after heat stress (Wang et al., 2018a,b). According to transcriptome and proteome analyses after heat stress, the protein level of LetrpE, a rate-limiting enzyme in the IAA biosynthesis pathway, in the thermotolerance strain S606 was increased by approximately 500-fold after heat stress, while its transcript level was obviously downregulated (Wang et al., 2018a). Further, LetrpE RNAi in S606 played a negative role in regulating L. edodes thermotolerance (Ma et al., 2018).

The objectives of the present study were as follows: (1) to analyze the structure of LeDnaJ07 in L. edodes thermotolerance strain S606 and stress-sensitive strain YS55; (2) to explore the effect of LeDnaJ07 on mycelial growth and resistance to heat stress in YS55; (3) to quantify the IAA content after $24 \mathrm{~h}$ of $38^{\circ} \mathrm{C}$ heat stress in sawdust medium; and (4) to verify the interaction between LeDnaJ07 and LetrpE via Y2H (yeast two-hybrid) and BiFC (bimolecular fluorescence complementation) analyses.

\section{MATERIALS AND METHODS}

\section{Strains and Culture Conditions}

L. edodes strain YS55 was used as the recipient host strain for gene overexpression and it was cultured at $25^{\circ} \mathrm{C}$ on $\mathrm{MYG}$ (malt yeast glucose) medium or sawdust medium. E. coli Trans1-T1 and Agrobacterium tumefaciens EHA105 were used for plasmid amplification and infecting L. edodes mycelia, respectively, and they were cultured in $50 \mu \mathrm{g} / \mathrm{ml}$ kanamycin Luria-Bertani (LB) medium.

\section{Extraction of RNA, LeDnaJ07 Cloning and Sequence Analysis}

Total RNA was extracted from L. edodes strain S606 and YS55 using the RNAiso Plus (TAKARA, Shanghai, China) method, and isolated RNA was then reverse-transcribed into cDNA using the HiScript II One Step RT-PCR Kit (Vazyme, Nanjing, China) according to the manufacturer's instruction. Based on L. eodes strain WX-1 genome information ${ }^{1}$ (Chen et al., 2016), the CDS (coding sequence) of LeDnaJ07 (Le01Gene01273) was amplified by PCR using L. edodes strain S606 and YS55 cDNA (primers listed in Supplementary Table S1), and the fragments were cloned into the pEASY-Blunt vector (TransGen Biotech, Beijing, China) for sequencing. The CDSs containing S606 and YS55 as well as WX-1 were aligned using ClustalW in MEGA 6.0, and the alignment result was visualized by GeneDoc. The protein sequences of LeDnaJ07 were aligned with $22 \mathrm{~J}$ proteins in Saccharomyces cerevisiae using the neighbor-joining method in MEGA 6.0.

\section{Vector Construction and Fungal Transformation}

The fungal overexpression vector was constructed as previously described (Ding et al., 2011). The Legpd (L. edodes glyceraldehyde-3-phosphate dehydrogenase) promoter was used to induce expression of hygromycin phosphotransferase, and the Leactin promotor was used to induce the mCherry fluorescent protein (mRFP) and LeDnaJ07. The coding sequences of mRFP and LeDnaJ07 were obtained by PCR using primers with the homologous arms containing restriction enzyme sites (EcoRI and KpnI) as well as pBlueScript SK plasmid and L. edodes CDNA as the template (Supplementary Table S1). The overexpression vector and control vector only containing mCherry protein were transferred into the $L$. edodes strain by $A$. tumefaciens infection. Nine transformants were selected randomly to analyze the overexpression efficiency of the transformants by qRT-PCR. mRFP expression was assessed by observing fluorescence using a fluorescence microscope, and the stable integration of the target gene into the genome of $L$. edodes was confirmed by Southern blot analysis (Yin et al., 2015). Two independent overexpression strains with the highest upregulation efficiency and one transformant (control strain) only containing mCherry protein were selected for further study.

\section{Mycelial Growth and Thermotolerance Susceptibility Assays}

After 10-day growth at $25^{\circ} \mathrm{C}$, the colony diameters of the transgenic and WT strains in the MYG and sawdust medium were measured to evaluate the effect of LeDnaJ07 on L. edodes mycelial growth. Moreover, the LeDnaJ07 over-expressed, control and WT strains were grown in plastic culture bags $(15 \mathrm{~cm}$

\footnotetext{
${ }^{1}$ http://legdb.chenlianfu.com/index.html
} 
in fold diameter and $30 \mathrm{~cm}$ in length) containing $0.8 \mathrm{~kg}$ (wet weight) of sterilized sawdust medium (78\% hardwood, $20 \%$ wheat bran, $1 \%$ lime, $1 \%$ gypsum and $55 \%$ water content). Five mycelial plugs ( $8 \mathrm{~mm}$ in diameter) were inoculated into each culture bag, and 12 culture bags were prepared separately for each of the transformant and wild-type (WT) strains. Culture bags were followed by culture at $25^{\circ} \mathrm{C}$ in the dark.

To test the effect of $\mathrm{LeDnaJ07}$ overexpression on the resistance of $L$. edodes against heat stress, thermotolerance assays, involving short- and long- term heat stresses, were performed according to our previous method (Wang et al., 2018b). In the short-term heat stress group, the stress temperature and recovery time were $38^{\circ} \mathrm{C}$ and $25 \mathrm{~d}$, respectively. Colony diameters and characteristics were used to quantify the sensitivity of the transgenic and WT strains to heat stress, with five replicates for each group.

\section{Detection and Measurement of IAA}

L. edodes mycelia treated or untreated by $38^{\circ} \mathrm{C}$ heat stress for $24 \mathrm{~h}$ were collected separately. To analyze IAA differences between transformant and WT strains, mycelial samples were grinded into fine powder in liquid nitrogen. IAA extraction and measurement methods were performed according to our previous study (Wang et al., 2018b).

\section{Yeast Two-Hybrid and Bimolecular Fluorescence Complementation Analyses}

The interaction between LeDnaJ07 with LetrpE was verified using the Matchmaker Gold Yeast Two-Hybrid (Y2H) System (Clontech, Palo Alto, CA, United States) (Yin et al., 2015). LeDnaJ07 and LetrpE were introduced into pDEST22 and pDEST32 plasmids as baits and preys, respectively. The bait and prey plasmids were co-transformed into $S$. cerevisiae strains MaV103 and MaV203 according to the manufacturer's instructions. Transformed yeast cells were assayed for growth onsynthetic dropout SD/-Trp-Leu-Ura plates, SD/-Trp-Leu-His plates and SD/-Trp-Leu plates containing X-a-galactosidase (Xa-gal) or 3-amino-1,2,4-triazole (3AT) (10 and $25 \mathrm{mM}$ ).

The full-length cDNA sequences of LeDnaJ07 and LetrpE were amplified by PCR and cloned into the BamHI/XhoI site of pSYCE-MR and pSCYNE-R vectors, respectively, generating CFPC-LeDnaJ07/LetrpE and LeDnaJ07/LetrpECFPN fusion constructs. The constructs were transformed into A. tumefaciens strain AGL-1. Transient expression of proteins in $N$. benthamiana leaves by $A$. tumefaciens infiltration was conducted as previously described (Kang et al., 2011; Yang et al., 2018). Cyan fluorescence of fusion proteins was assayed 2-3 d after infiltration using a Zeiss LSM510 with excitation/emission wavelengths of 405 and $477 \mathrm{~nm}$, respectively.

\section{Statistical Analysis}

All data averaged from the five independent sample measurements were used to ensure the reliability and reproducibility of the change trend between WT and overexpression strains. The significance of the differences between the analyzed samples was determined by oneway analysis of variance (ANOVA) and tested for significant $(P<0.05)$ treatment differences using Duncan's multiple range test.

\section{RESULTS}

\section{Analysis of LeDnaJ07 CDS in Different L. edodes Strains}

The CDS difference of LeDnaJ07 between heat-resistant strain S606, stress-sensitive strain YS55 and cultivation strain WX1 was analyzed using the PCR sequecing result. According to the prediction of DnaJ domain in https://www.ncbi.nlm.nih.gov/
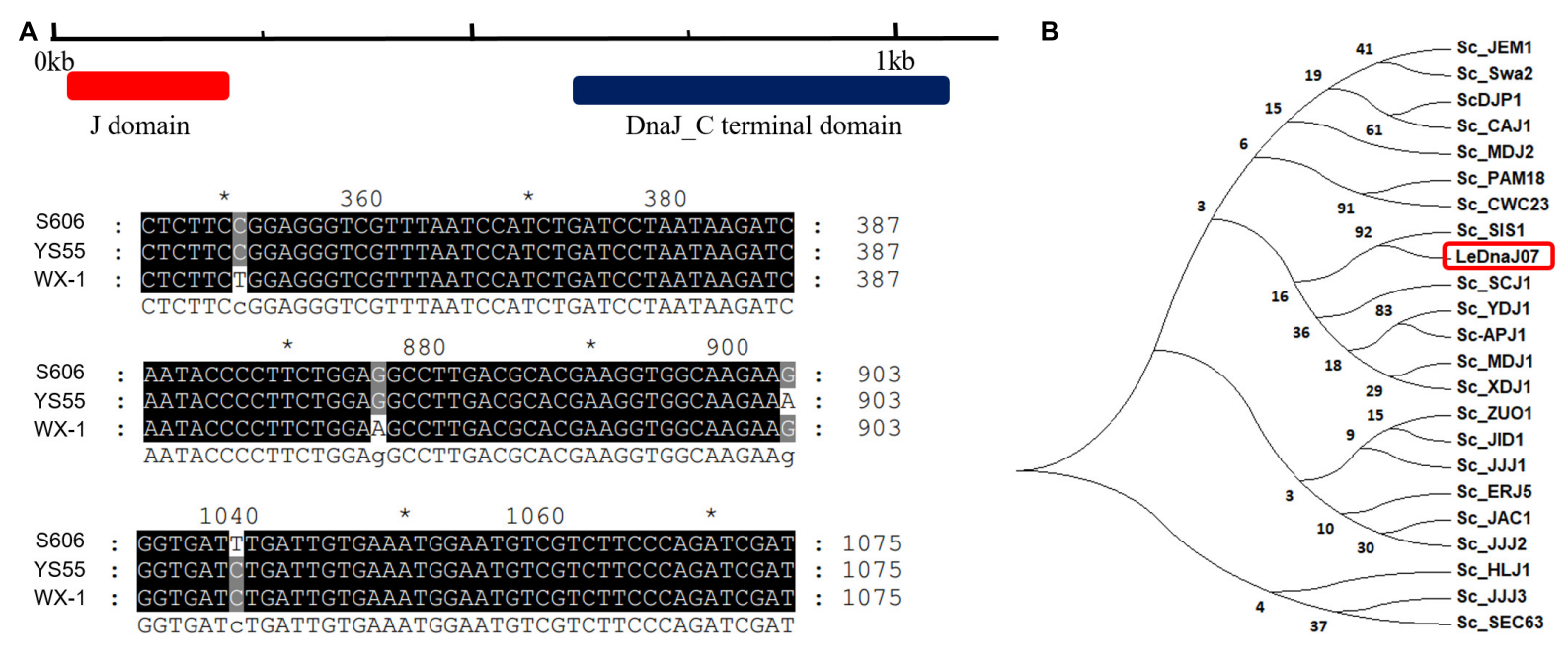

FIGURE 1 | LeDnaJ07 sequence analysis. (A) Structure analysis and sequence alignment of LeDnaJ07 CDS in three L. edodes strains. (B) Molecular phylogenetic tree of LeDnaJ07 generated with the neighbor-joining (NJ) method using MEGA 7.0. An unrooted phylogenetic tree was generated based on the alignment of the amino acid (AA) sequences containing LeDnaJ07 and $22 \mathrm{~S}$. cerevisiae J proteins. 

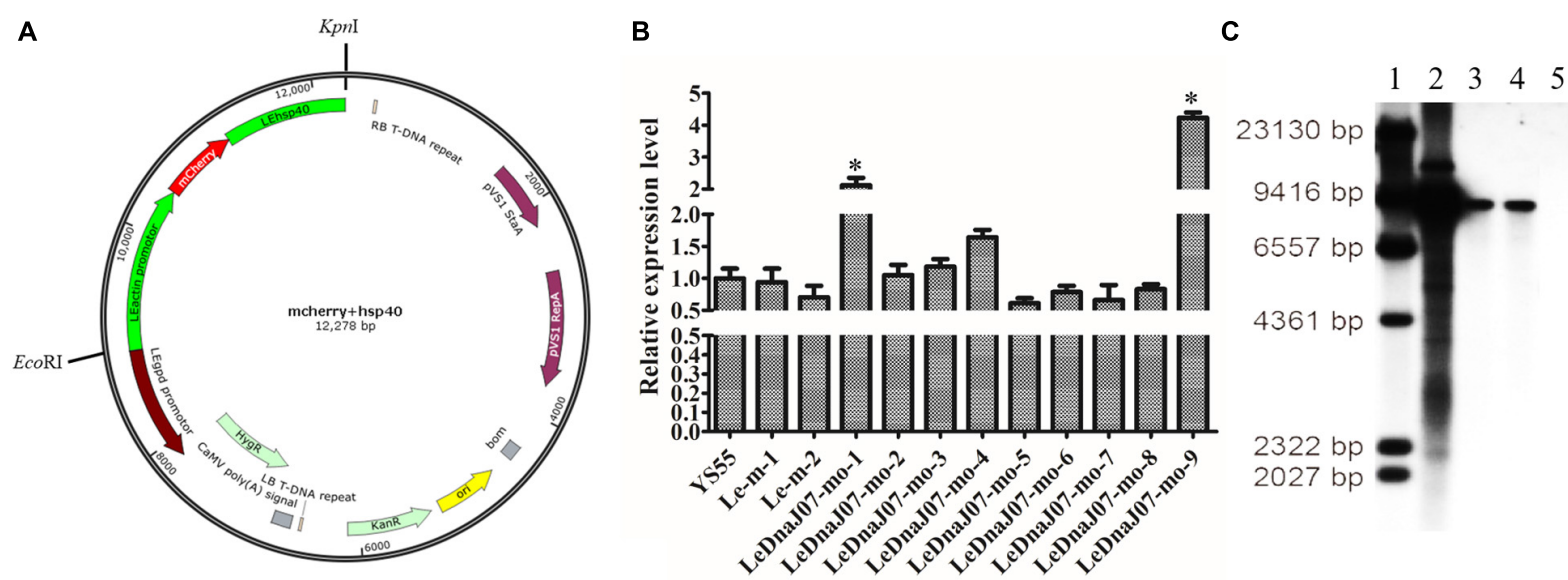

FIGURE 2 | Vector map and identification of LeDnaJ07 overexpression transformants. (A) Vector map of LeDnaJ07 overexpression. (B) qRT-PCR analysis of LeDnaJ07 overexpression transformants. YS55, wild type strain; Le-m-1/2, Control transgenic strains; LeDnaJ07-mo-1-9, LeDnaJ07 overexpression strains; *, represents the significant difference. (C) Southern blot analysis of genomic DNA isolated from the transformants and WT strain digested with HindllI. Lane 1-5, Maker, pCAMBIA1300-g plasmid, LeDnaJ07-mo-1, LeDnaJ07-mo-9, and the WT strain YS55, respectively.
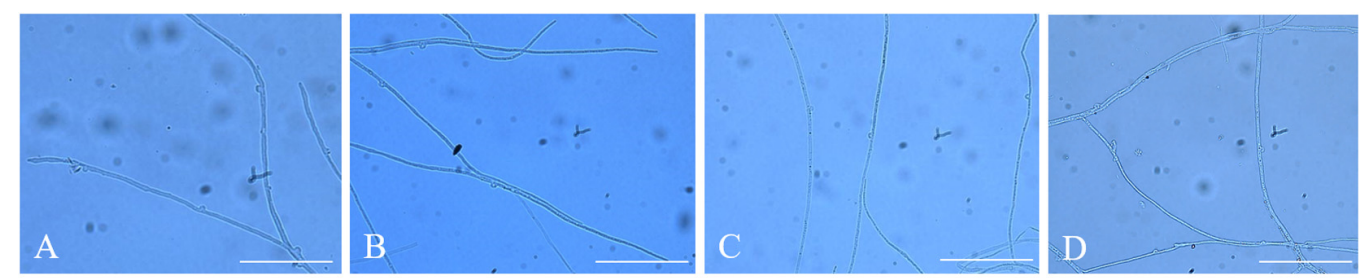

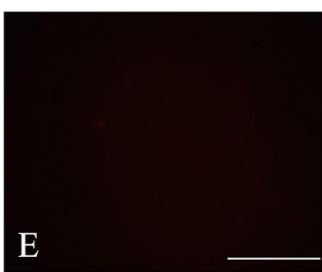

YS55

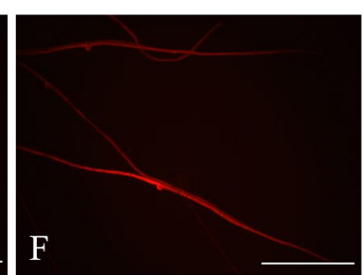

LeDnaJ07-mo-1

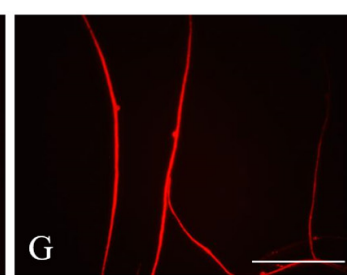

LeDnaJ07-mo-9

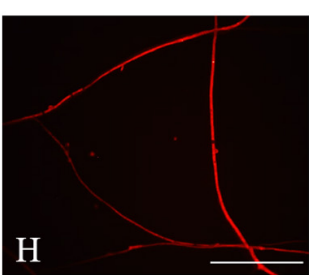

Le-m-1

FIGURE 3 | Expression of mCherry in the LeDnaJ07 overexpression transformant, control transgenic and WT strains. (A-D) Detection of L. edodes mycelia without fluorescence. (E-H) Detection of $L$. edodes mycelia with fluorescence under red light. Bar, $10 \mu \mathrm{m}$.

Structure/cdd/wrpsb.cgi, J domain located from 10 to $198 \mathrm{bp}$ in LeDnaJ07 CDS (Figure 1A). In terms of sequence alignment, it was found that only four of 1119 base pairs out of J domain exhibited a difference among three $L$. edodes strains (Figure 1A and Supplementary Figure S1), which made no changes on the amino acid sequence of three LeDnaJ07 proteins. This result indicated that LeDnaJ07 proteins in different L. edodes strains were identical. As shown in Figure 1B, LeDnaJ07 exhibited a 92\% similarity to SIS1 in S. cerevisiae, indicating that LeDnaJ07 could play an important role during translation initiation.

\section{Construction of LeDnaJ07 Overexpression Strains}

The overexpression vector pCAMBIA1300-g-mo was used to express the fusion protein mRFP-LeDnaJ07, and the hygromycin
$B$ resistance gene in that overexpression vector was treated as the selectable marker (Figure 2A). Nevertheless, the vector pCAMBIA1300-g-m only expressing the mRFP was treated the contorl vector. After introducing the expression vector and the control vector into the mycelia of $L$. edodes via $A$. tumefaciens mediation, the expression level of LeDnaJ07 was used to confrim the transformants. Among all the transformants, two overexpression transformants LeDnaJ07-mo-1 and LeDnaJ07mo-9 had an about two and four-fold upregulation $(P<0.05)$, respectively, but no significant expression changes found in other transformatns and two control transformants Le-m-1 and Lem-2 (Figure 2B). Southern blot showed that one copy of the insert fragment was found in overexpression strains LeDnaJ07mo-1 and LeDnaJ07-mo-9, but no copy in the WT strain (Figure 2C), indicating the integration of the insert fragment including in LeDnaJ07 gene into L. edodes genome. To verify 

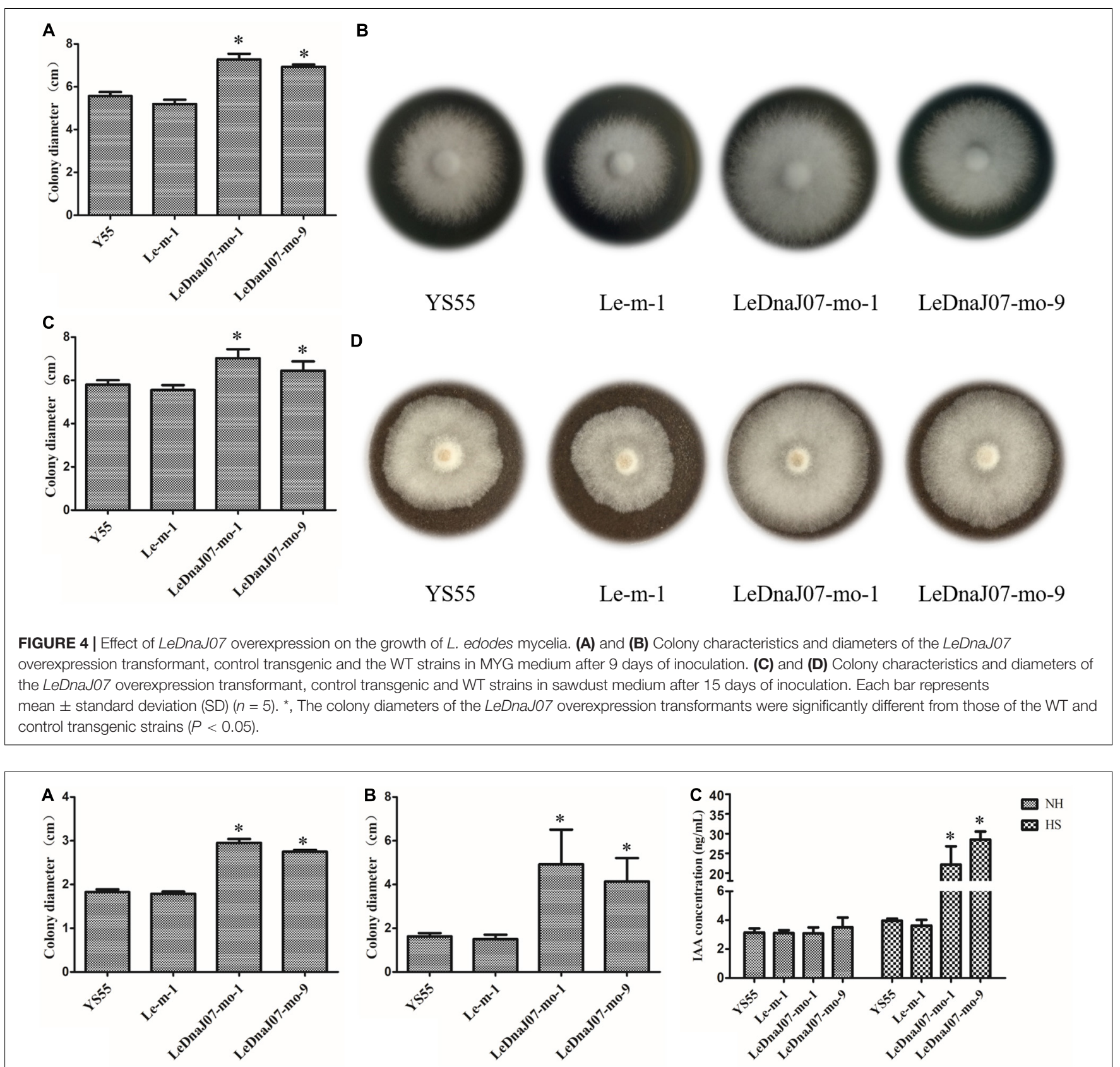

FIGURE 5 | LeDnaJ07 overexpression enhances L. edodes thermotolerance and IAA biosynthesis after heat stress. (A) Colony diameters of the WT, control transgenic and LeDnaJ07 overexpression transformant strains grown in sawdust medium for 15 days of recovery from the 15 days of $31^{\circ} \mathrm{C}$ heat stress. (B) Colony diameters of the WT, control transgenic and LeDnaJ07 overexpression transformant strains in sawdust medium after 25 days of recovery from the $24 \mathrm{~h}$ of $38^{\circ} \mathrm{C}$ heat stress. (C) IAA concentration change in the wild type YS55 and LeDnaJ07 overexpression transformants after $38^{\circ} \mathrm{C}$ heat stress for 24 h. Each bar represents mean \pm standard deviation $(\mathrm{SD})(n=5)$. $\mathrm{NH}$, not heat stress. $\mathrm{HS}$, heat stress. ${ }^{*}$, significant difference $(P<0.05)$.

the expression level of the introduced mCherry reporter gene, mycelia fluorescence of three individual transformants grown on MYG medium were detected using a fluorescence microscope. As shown in Figure 3, the clear distribution of red fluorescence was found in the mycelia of three individual transformants. These results suggested that the LeDnaJ07 overexpression recombinants were successfully obtained.

\section{Overexpression of LeDnaJ07 Enhances the Mycelial Growth of $L$. edodes}

As shown in Figure 4, in MYG medium, the colony diameters of the two overexpression transformants (LeDnaJ07-mo-1 and LeDnaJ07-mo-9) were 7.28 and $6.93 \mathrm{~cm}$ after 9 days of inoculation, with 5.57 and $5.20 \mathrm{~cm}$ diameters for the control and WT strains (Figures $4 \mathbf{4 A , B}$ ); and the colony 


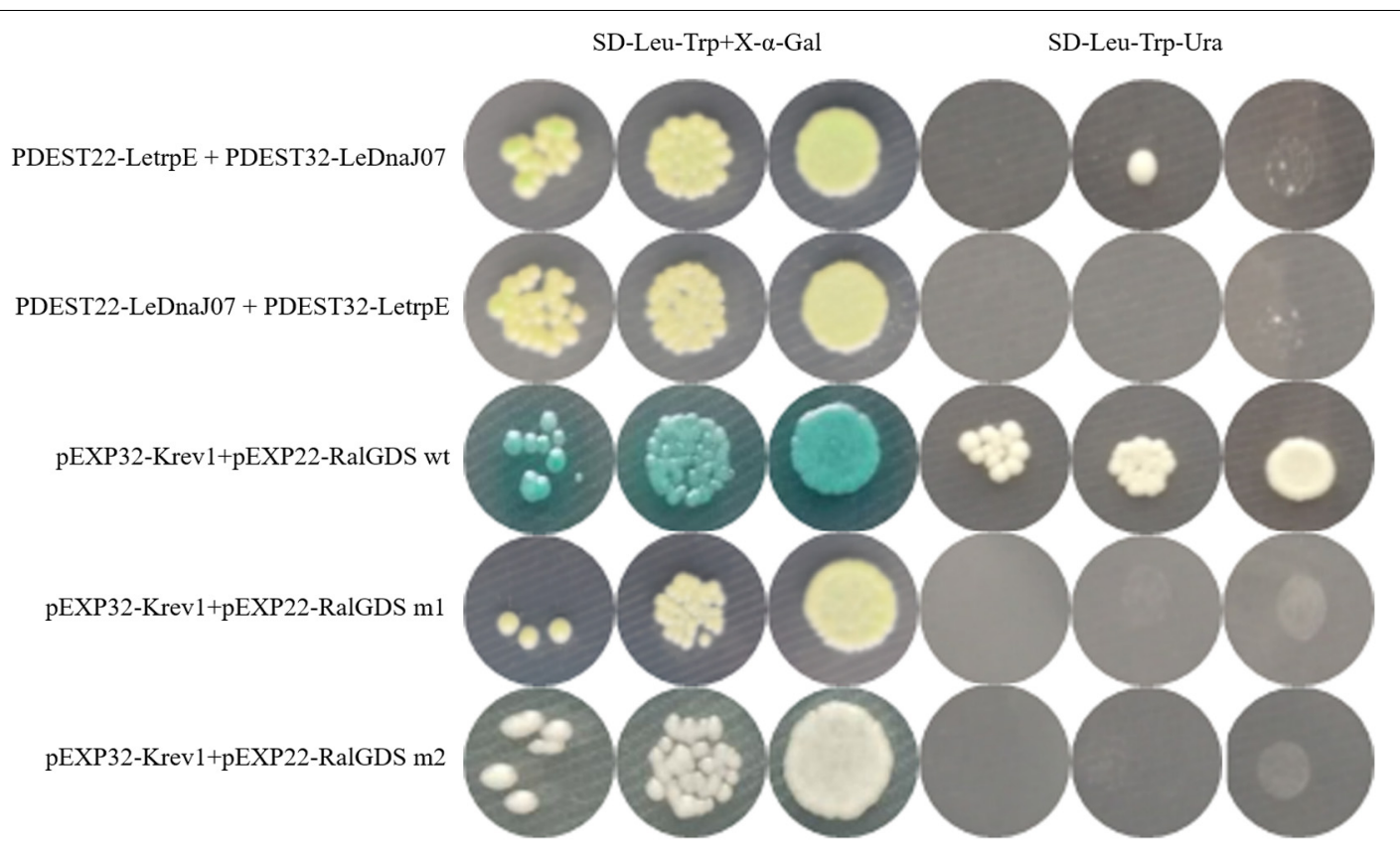

FIGURE 6 | Interaction verification of LeDnaJ07 with LetrpE in the Y2H system on the plates with SD/-Leu-Trp and SD/-Leu-Trp-Ura. Tested groups, DEST22-LeDnaJ07 + PDEST32-LetrpE, and PDEST22-LetrpE + PDEST32-LeDnaJ07; Positive control, pEXP32-Krev1 + pEXP22-RalGDS wt; Weak position interaction control, pEXP32-Krev1 + pEXP22-RalGDS m1; Negative control, pEXP32-Krev1 + pEXP22-RalGDS m2.

PDEST22-LetrpE + PDEST32-LeDnaj07
pEXP32-Krev1+pEXP22-RalGDS wt
pEXP32-Krev1+pEXP22-RalGDS m2
FIGURE 7 I Interaction verification of LeDnaJ07 with LetrpE in the Y2H system on the plates with SD/-Leu-Trp-His and SD/-Leu-Trp and different concentration 3AT.
Tested groups, DEST22-LeDnaJ07 + PDEST32-LetrpE and PDEST22-LetrpE + PDEST32-LeDnaJ07; Positive control, pEXP32-Krev1 + pEXP22-RalGDS wt; Weak
position interaction control, pEXP32-Krev1 + pEXP22-RalGDS m1; Negative control, pEXP32-Krev1 + pEXP22-RalGDS m2.

diameters of the two overexpression transformants were 7.03 and $6.45 \mathrm{~cm}$ after inoculation for 15 days in sawdust medium, with diameters of 5.80 and $5.55 \mathrm{~cm}$ for the control and WT strain (Figures 4C,D). Besides, it was documented that the mycelia of LeDnaJ07 over-expressed transformants were full of the cultivation bags after 45 days of growth, while the mycelia of WT strain YS55 and control strain Le-m-1 covered $70 \%$ of the cultivation bags (not shown), indicating that improving the expression of LeDnaJ07 could promote the growth of $L$. edodes mycelia in MYG and sawdust medium.

\section{LeDnaJ07 Overexpression Is of Benefit to $L$. edodes Thermotolerance}

The effect of LeDnaJ07 on L. edodes thermotolerance was estimated by comparing the colony diameters after heat stress among WT, control and LeDnaJ07 overexpression transformant strains. Long-term heat stress lasted for 15 days at $31^{\circ} \mathrm{C}$, and short-term heat stress lasted $24 \mathrm{~h}$ at $38^{\circ} \mathrm{C}$. From the perspective of the thermotolerance assays, no growth was observed in the mycelia of all strains after $31^{\circ} \mathrm{C}$ heat stress for 15 days. Nevertheless, after 15 days of recovery at $25^{\circ} \mathrm{C}$, only the mycelia 


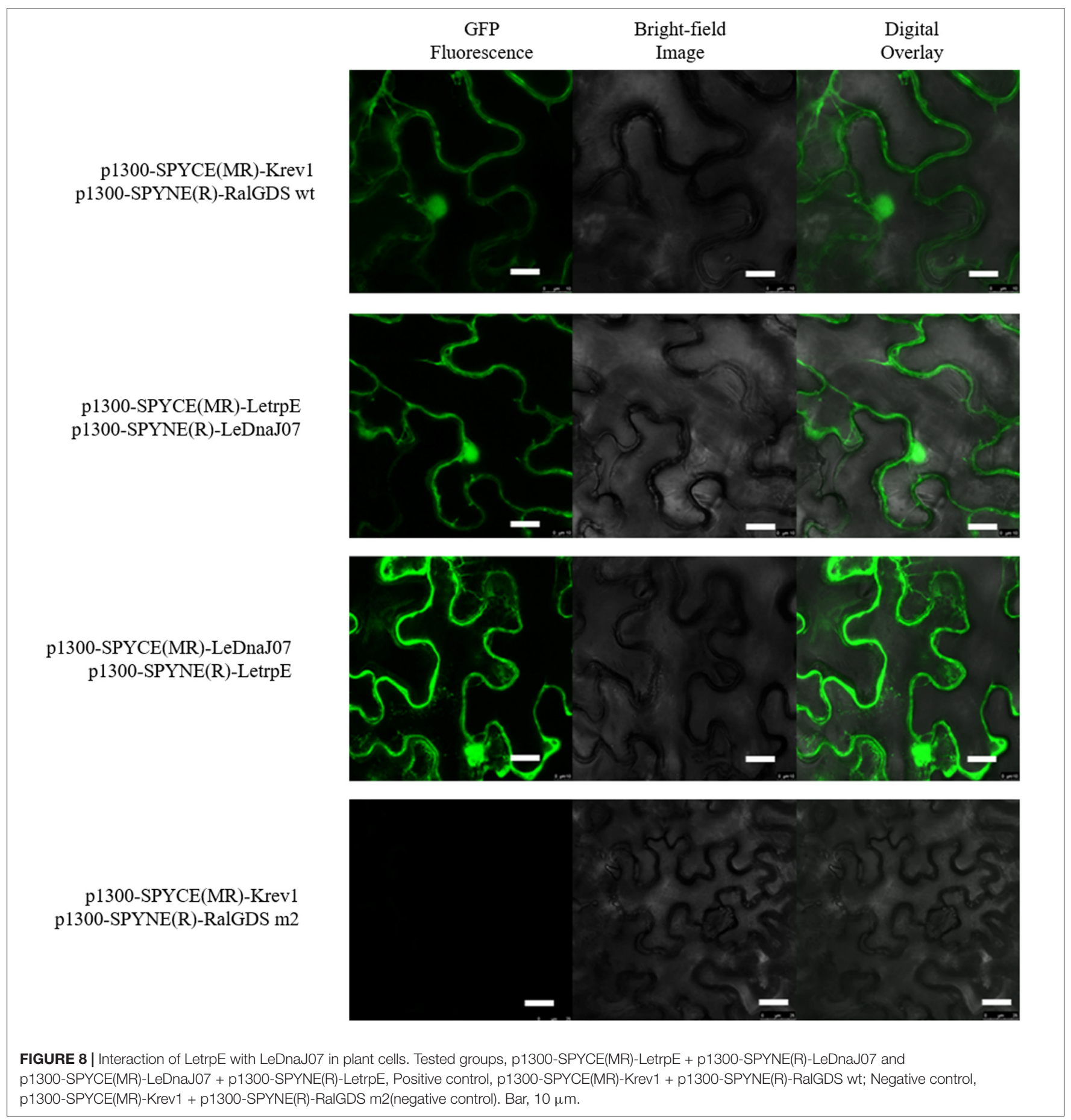

of the two LeDnaJ07 overexpressed transformants with a colony diameter about $3 \mathrm{~cm}$ regrew at $31^{\circ} \mathrm{C}$ heat stress, whereas an about $2 \mathrm{~cm}$ colony diameter was found in WT and control transgenic strains (Figure 5A). After $24 \mathrm{~h}$ of heat stress at $38^{\circ} \mathrm{C}$, the mycelia of the two overexpression transformants showed a colony diameter about $4.5 \mathrm{~cm}$ after a 25 -day recovery at $25^{\circ} \mathrm{C}$, but the mycelia of control transgenic and WT strains showed a colony diameter of $2.0 \mathrm{~cm}$ and were dead and dissolved (Figure $5 \mathbf{B}$ ). The similar results of the long-term and short-term heat stress assays demonstrated that LeDnaJ07 promoted the recovery capacity and thermotolerance of $L$. edodes mycelia in response to heat stress.

\section{The Overexpression of LeDnaJ07 Promotes Intracellular IAA Biosynthesis After Heat Stresses}

At $25^{\circ} \mathrm{C}$, the intracellular IAA concentrations of YS55, Le-m-1 and the two LeDnaJ07 overexpression strains displayed a slight 
difference, but they showed significant differences after heat stress (Figure 5C). After $24 \mathrm{~h}$ of heat stress at $38^{\circ} \mathrm{C}$, the intracellular IAA concentration had a 7.16 and 8.12 -fold increase in the two LeDnaJ07 overexpression transformants, but only a 1.26 and 1.17-fold increase in the WT strain YS55 and control transgenic strain Le-m-1, indicating that LeDnaJ07 was beneficial to IAA biosynthesis during the response to heat stress.

\section{Interaction Between LeDnaJ07and LetrpE in Yeast and Tobacco Leaf Cells}

According to the result of self-activation assay, it was found that the yeast cells only containg one of LeDnaJ07 and LetrpE could grow ont SD/-Trp plates, but no growth found on $\mathrm{SD} /$-Trp-Leu and SD/-Trp-Ade plates, suggesting that they showed no self-activation effect in transgenic yeast cells (Supplementary Figure S2). In the $\mathrm{Y} 2 \mathrm{H}$ assay, after 3 days of culture at $30^{\circ} \mathrm{C}$, yeasts from all mixtures grew on SD/-TrpLeu plates (Figure 6). The yeasts from mixture containing the positive control (pEXP32-Krev1 + pEXP22-RalGDS wt) turned blue, and the yeasts from the weak positive interaction control (pEXP32-Krev1 + pEXP22-RalGDS m1) and the tested group (PDEST22-LeDnaJ07 + PDEST32-LetrpE and PDEST22LetrpE + PDEST32-LeDnaJ07) exhibited light blue. Whereas, the yeasts from the negative control (pEXP32-Krev1 + pEXP22RalGDS m2) displayed white. In addition, the mated yeasts from the positive control and tested groups exhibited the growth on SD/-Trp-Leu-Ura plates (Figure 6). Except for the negative control, the mated yeasts of other four groups grew on SD/-LeuTrp plates with 10 and $25 \mathrm{mM}$ 3AT (Figure 7). The above results indicated that a weak interaction occurred between LeDnaJ07 and LetrpE. Furthermore, as shown in Figure 8, the BiFC assay showed that cells from the positive control (p1300-SPYCE(MR)Krev1 + p1300-SPYNE(R)-RalGDS wt) and tested groups (p1300-SPYCE(MR)-LeTrpE + p1300-SPYNE(R)-LeDnaJ07 and p1300-SPYNE(R)-LetrpE + p1300-SPYCE(MR)-LeDnaJ07) exhibited green fluorescence, but no green fluorescence was observed in the negative group (p1300-SPYCE(MR)Krev1 + p1300-SPYNE(R)-RalGDS m2). Taken together, these data verified that LeDnaJ07 had an interaction with LetrpE.

\section{DISCUSSION}

Numerous studies have shown that DnaJ proteins are involved in biotic and abiotic stresses for human and plants (Shen and Yu, 2011; Kakkar et al., 2012; Lee et al., 2018; Zhong et al., 2018). However, the regulation of the DnaJs on the resistance of higher basidiomycetes to biotic and abiotic stresses remains poorly understood. According to our previous studies, it was documented that the RNAi of the LeDnaJ07 decreased mycelial growth and the resistance to heat stress, while LeDnaJ07 overexpression enhanced the thermotolerance of $L$. edodes heatresistant strain S606 (Wang G.Z. et al., 2016, Wang et al., 2018b). However, it is worthy of study whether LeDnaJ07 overexpression regulates the resistance of other $L$. edodes strains to stresses or not, how LeDnaJ07 regulates the response of L. edodes to thermotolerance. In this study, we intended to provide evidence

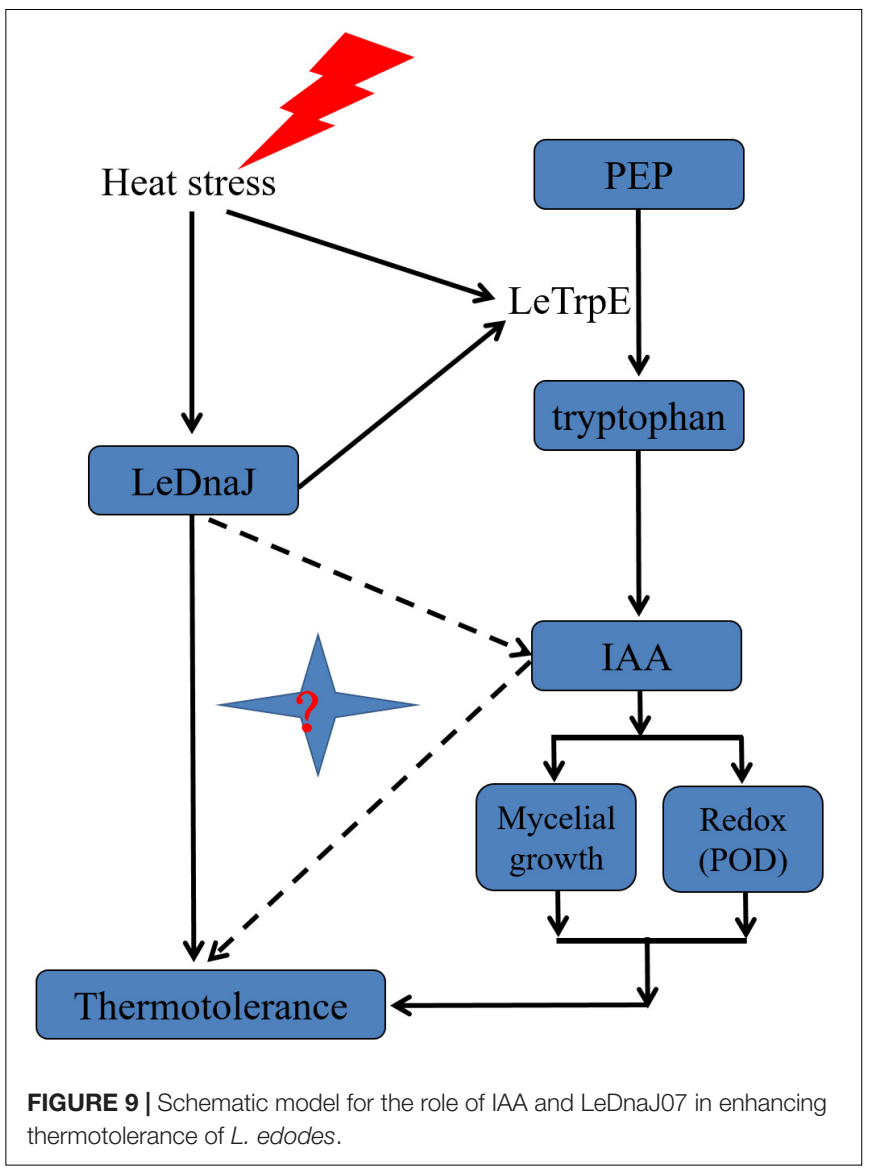

that J domain in different $L$. edodes strains was consistent, and the overexpression of the chaperone LeDnaJ07 participated in regulating thermotolerance and mycelial growth by targeting LetrpE in the stress-sensitive strain YS55.

In plant, a large number of papers reported that DnaJ proteins regulated growth and development. For Arabidopsis thaliana and rice, DnaJ protein loss led to an obvious late-flowering trait and largely abnormal cellular structures (Shen and Yu, 2011; Zhu et al., 2015). Overexpression of BIL2, one gene encoding the mitochondrial DnaJ protein, promoted elongation of $A$. thaliana cells by promoting ATP synthesis (Bekhochir et al., 2013). Silencing of PSA2, which encodes DnaJ family proteins, led to an obvious decrease in chlorophylls and total carotenoids, further variegated leaves and retarded growth (Wang Y.W. et al., 2016). The present study documented that colony diameters of two overexpression transformants were significantly larger than them of the control transgenic and WT strains on sawdust and MYG medium at the same culture condition (Figure 4), indicating that LeDnaJ07 overexpression exhibited a positive role in regulating $L$. edodes mycelial growth and development. These results were consistent with the results of our previous study that LeDnaJ07 silence resulted in a dramatically retarded mycelial growth in the heat-resistant strain S606 (Wang et al., 2018b). Nevertheless, it is still unknown how LeDnaJ07 overexpression promotes mycelial growth. 
Furthermore, an increasing number of reports have demonstrated that the high expression and accumulation of DnaJ proteins positively regulated the thermotolerance of plants. For example, the double knock-out of AtDjA2 and AtDjA3 impaired the thermotolerance of $A$. thaliana young seedlings, whereas overexpression of both genes increased the thermotolerance of transgenic plants ( $\mathrm{Li}$ et al., 2007). In transgenic tomatoes, DnaJ protein overexpression facilitated heat tolerance by regulating ROS and $\mathrm{H}_{2} \mathrm{O}_{2}$ and protected Rubisco activity from degradation under heat stress by keeping the levels of proteolytic enzymes low (Kong et al., 2014a; Wang et al., 2015, 2019). In the present study, the mycelia of the two LeDnaJ07 overexpression strains regrew after 15 -day of heat stress at $31^{\circ} \mathrm{C}$ and $24 \mathrm{~h}$ of heat stress at $38^{\circ} \mathrm{C}$, but no growth was observed in the control transgenic and WT strains at these conditions (Figures $5 \mathbf{A}, \mathbf{B}$ ). In addition, we observed that mycelia of the control transgenic and WT strains were dissolved after $38^{\circ} \mathrm{C}$ heat stress, whereas the overexpression strains were mildly affected by heat stress. This difference in the phenotype trait of the test $L$. edodes strains in response to heat stress demonstrated that the LeDnaJ07 overexpression alleviated heat stress, providing further evidence that DnaJ proteins can enhance the thermotolerance in different L. edodes strains.

The present study also showed that overexpression of LeDnaJ07 in stress-sensitive strain YS55 promoted the IAA biosynthesis under heat stress (Figure 5C), which is consistent with our previous studies. LeDnaJ07 RNAi and overexpression decreased and promoted, respectively, the intracellular IAA concentration after heat stress, and $0.01 \mathrm{mM}$ IAA partly restored the resistance of LeDnaJ07 RNAi strains to heat stresses (Wang G.Z. et al., 2016, Wang et al., 2018b), indicating that LeDnaJ07 is quite important for IAA biosynthesis under heat stress condition. Anthranilate synthase TrpE is a key ratelimiting enzyme in the tryptophan-dependent IAA biosynthesis pathway (Ishimoto et al., 2010). In our study, the yeast twohybrid system and BiFC assays proved that LeDnaJ07 interacts with L. edoeds LetrpE (Figures 6-8), suggesting that the LeDnaJ07/LetrpE machinery possibly may play a significant role in thermotolerance by regulating IAA biosynthesis. According to our previous study, exogenous IAA and its analoges may alleviate the effect of oxidative damage induced by heat stress on the mycelia of $L$. edodes by regulating the expression of redox enzymes, such as SOD and LOX (Zhou et al., 2018). These results demonstrated that LeDnaJ07 overexpression enhanced the extracellular IAA content by interacting with LetrpE (a rate-limiting enzyme in the IAA biosynthesis pathway), and then improved the thermotolerance of $L$. edodes mycelia by mediating redox enzymes.

\section{REFERENCES}

Bekhochir, D., Shimada, S., Yamagami, A., Kanda, S., Ogawa, K., Nakazawa, M., et al. (2013). A novel mitochondrial DnaJ/Hsp40 family protein BIL2 promotes plant growth and resistance against environmental stress in brassinosteroid signaling. Planta 237, 1509-1525. doi: 10.1007/s00425-013-1859-3

Cao, X. T., Bian, Y. B., Xiao, X. J., and Wang, G. Z. (2015). Effect of heat stress on Lentinula edodes mycelial growth recovery and resistance to Trichoderma harzianum. Acta Edulis Fungi 22, 81-85.

\section{CONCLUSION}

In summary, we cloned and analyzed the LeDnaJ07 gene in L. edodes heat-resistant strain S606 and stress-sensitive strain YS55. Overexpression of LeDnaJ07 in YS55 enhanced the tolerance to heat stress in the transgenic strains, with an obvious increase in mycelial growth and intracellular IAA concentration in the transgenic strains compared with the control transgenic and WT strains. In addition, the present study for the first time showed that LeDnaJ07 interacted with LetrpE. We constructed a model for the role of LeDnaJ07 and the IAA signal pathway in enhancing L. edodes thermotolerance (Figure 9). This study provides valuable information for the relative expression level of DnaJ proteins in stress-resistant mushroom breeding. Further study will focus on the mechanism by which LeDnaJ07 regulates L. edodes thermotolerance via the IAA signal pathway.

\section{DATA AVAILABILITY STATEMENT}

All datasets generated for this study are included in the article/Supplementary Material.

\section{AUTHOR CONTRIBUTIONS}

GW, YZ, HK, YX, YB, and YG conceptualized the study. CW helped with the data curation. GW, YL, and YG contributed to the formal analysis. $\mathrm{YB}$ was responsible for the funding acquisition. YL and CW carried out the investigation. GW, YL, $\mathrm{CM}, \mathrm{HK}$, and YG worked on the methodology. YX contributed to the resources. GW, YL, and $\mathrm{CW}$ were responsible for the validation. GW, YL, and CM helped with the visualization. GW wrote the original draft. YZ, YB, and YG reviewed and edited the manuscript.

\section{FUNDING}

This research was funded by the National Natural Science Foundation of China (Grant Nos. 31672211 and 31972476).

\section{SUPPLEMENTARY MATERIAL}

The Supplementary Material for this article can be found online at: https://www.frontiersin.org/articles/10.3389/fmicb. 2020.00707/full\#supplementary-material

Caplan, A. J., Tsai, J., Casey, P. J., and Douglas, M. G. (1992). Farnesylation of YDJ1p is required for function at elevated growth temperatures in Saccharomyces cerevisiae. J. Biol. Chem. 267, 18890-18895.

Chang, S. (2005). Witnessing the development of the mushroom industry in China. Acta Edulis Fungi 12, 3-19.

Chen, L., Gong, Y., Cai, Y., Liu, W., Zhou, Y., Xiao, Y., et al. (2016). Genome sequence of the edible cultivated mushroom Lentinula edodes (Shiitake) reveals insights into lignocellulose degradation. Plos One 11:e0160336. doi: 10.1371/ journal.pone.0160336 
Deng, L., Lu, Z., Shen, Y., and Song, S. (2014). Identification thermotolerance function of hsp20 and Adcs genes from Agaricus bisporus by Arabidopsis thaliana. J. Xiamen Univ. 53, 267-272.

Ding, Y., Liang, S., Lei, J., Chen, L., Kothe, E., and Ma, A. (2011). Agrobacterium tumefaciens mediated fused egfp-hph gene expression under the control of gpd promoter in Pleurotus ostreatus. Microbiol. Res. 166, 314-322. doi: 10.1016/j. micres.2010.07.001

Finimundy, T. C., Dillon, A. J. P., Henriques, J. A. P., and Ely, M. R. (2014). A review on general nutritional compounds and pharmacological properties of the Lentinula edodes mushroom. Food Nutr. Sci. 5:1095. doi: 10.4236/fns.2014. 512119

Ishimoto, M., Rahman, S. M., Hanafy, M. S., Khalafalla, M. M., El-Shemy, H. A., Nakamoto, Y., et al. (2010). Evaluation of amino acid content and nutritional quality of transgenic soybean seeds with high-level tryptophan accumulation. Mol. Breeding 25, 313-326. doi: 10.1007/s11032-009-9334-3

Kakkar, V., Prins, L. C. B., and Kampinga, H. H. (2012). DNAJ proteins and protein aggregation diseases. Curr. Top. Med. Chem. 12, 2479-2490. doi: 10.2174/ 1568026611212220004

Kang, H., Zhu, H., Chu, X., Yang, Z., Yuan, S., Yu, D., et al. (2011). A novel interaction between CCaMK and a protein containing scythe_N Ubiquitin-like domain in Lotus japonicus. Plant Physiol. 155, 1312-1324. doi: 10.1104/pp.110. 167965

Kong, F., Deng, Y., Wang, G., Wang, J., Liang, X., and Meng, Q. (2014a). LeCDJ1, a chloroplast DnaJ protein, facilitates heat tolerance in transgenic tomatoes. J. Integrat. Plant Biol. 56, 63-74. doi: 10.1111/jipb.12119

Kong, F., Deng, Y., Zhou, B., Wang, G., Wang, Y., and Meng, Q. (2014b). A chloroplast-targeted DnaJ protein contributes to maintenance of photosystem II under chilling stress. J. Exp. Bot. 65, 143-158. doi: 10.1093/jxb/ert357

Kong, W., Huang, C., Chen, Q., Zou, Y., and Zhang, J. (2012a). Nitric oxide alleviates heat stress-induced oxidative damage in Pleurotus eryngii var. tuoliensis. Fungal Genet. Biol. 49, 15-20. doi: 10.1016/j.fgb.2011. 12.003

Kong, W., Huang, C., Chen, Q., Zou, Y., Zhao, M., and Zhang, J. (2012b). Nitric oxide is involved in the regulation of trehalose accumulation under heat stress in Pleurotus eryngii var. tuoliensis. Biotechnol. Lett. 34, 1915-1919. doi: 10.1007/ s10529-012-0988-2

Lee, K. W., Rahman, M. A., Kim, K. Y., Choi, G. J., Cha, J. Y., Cheong, M. S., et al. (2018). Overexpression of the alfalfa DnaJ-like protein (MsDJLP) gene enhances tolerance to chilling and heat stresses in transgenic tobacco plants. Turk. J. Biol. 42, 12-22. doi: 10.3906/biy-1705-30

Li, G. L., Chang, H., Li, B., Zhou, W., Sun, D. Y., and Zhou, R. G. (2007). The roles of the atDjA2 and atDjA3 molecular chaperone proteins in improving thermotolerance of Arabidopsis thaliana seedlings. Plant Sci. 173, 408-416. doi: 10.1016/j.plantsci.2007.06.013

Liu, Y. N., Zhang, T. J., Lu, X. X., Ma, B. L., Ren, A., Shi, L., et al. (2017). Membrane fluidity is involved in the regulation of heat stress induced secondary metabolism in Ganoderma lucidum. Environ. Microbiol. 19, 1653-1668. doi: $10.1111 / 1462-2920.13693$

Liu, R., Shi, L., Zhu, T., Yang, T., Ren, A., Zhu, J., et al. (2018). Cross talk between nitric oxide and calcium-calmodulin regulates ganoderic acid biosynthesis in Ganoderma lucidum under heat stress. Appl. Environ. Microbiol. 84:e043-18. doi: 10.1128/AEM.00043-18

Lu, Z., Kong, X., Lu, Z., Xiao, M., Chen, M., Zhu, L., et al. (2014). Para-aminobenzoic acid (PABA) synthase enhances thermotolerance of mushroom Agaricus bisporus. PLoS One 9:e91298. doi: 10.1371/journal.pone.00 91298

Ma, C. J., Wang, G. Z., Zhou, S. S., Luo, Y., Gong, Y. H., and Bian, Y. B. (2018). Functional analysis of anthranilate synthase gene LetrpE in Lentinula edodes by RNAi mediated gene knockdown. Mycosystema 37, 581-588.

Qiu, Z., Wu, X., Zhang, J., and Huang, C. (2017). High temperature enhances the ability of Trichoderma asperellum to infect Pleurotus ostreatus mycelia. PLoS One 12:e0187055. doi: 10.1371/journal.pone.0187055

Rampuria, S., Bag, P., Rogan, C. J., Sharma, A., Gassmann, W., and Kirti, P. B. (2018). Pathogen-induced AdDjSKI of the wild peanut, Arachis diogoi, potentiates tolerance of multiple stresses in E. coli and tobacco. Plant Sci. 272, 62-74. doi: 10.1016/j.plantsci.2018.03.033
Shen, L., and Yu, H. (2011). The J-domain protein J3 mediates the integration of flowering signals in Arabidopsis. Plant Cell 23, 499-415. doi: 10.1105/tpc.111. 083048

Suetsugu, N., Kagawa, T., and Wada, M. (2005). An auxilin-like J-domain protein, JAC1, regulates phototropin-mediated chloroplast movement in Arabidopsis. Plant Physiol. 139, 151-162. doi: 10.1104/pp.105.067371

Wang, G., Cai, G., Xu, N., Zhang, L., Sun, X., Guan, J., et al. (2019). Novel DnaJ protein facilitates thermotolerance of transgenic tomatoes. Int. J. Mol. Sci. 20:367. doi: 10.3390/ijms20020367

Wang, G., Kong, F., Zhang, S., Meng, X., Wang, Y., and Meng, Q. (2015). A tomato chloroplast-targeted DnaJ protein protects Rubisco activity under heat stress. J. Exp. Bot. 66, 3027-3040. doi: 10.1093/jxb/erv102

Wang, G. Z., Cao, X. T., Ma, X. L., Guo, M. P., Liu, C. H., Yan, L. L., et al. (2016). Diversity and effect of Trichoderma spp. associated with green mold disease on Lentinula edodes in China. Microbiol. Open 5, 709-718. doi: 10.1002/mbo3.364

Wang, Y. W., Chen, S. M., Wang, W. J., Huang, X. Q., Zhou, C. F., Zhuang, Z., et al. (2016). The DnaJ-like zinc finger domain protein PSA2 affects light acclimation and chloroplast development in Arabidopsis thaliana. Front. Plant Sci. 7:360. doi: 10.3389/fpls.2016.00360

Wang, G. Z., Ma, C. J., Luo, Y., Zhou, S. S., Zhou, Y., Ma, X. L., et al. (2018a). Proteome and transcriptome reveal involvement of heat shock proteins and indoleacetic acid metabolism process in Lentinula edodes thermotolerance. Cell Physiol. Biochem. 50, 1617-1637. doi: 10.1159/000494784

Wang, G. Z., Zhou, S. S., Luo, Y., Ma, C. J., Gong, Y. H., Zhou, Y., et al. (2018b). The heat shock protein 40 LeDnaJ07 regulates stress resistance and indole-3acetic acid biosynthesis in Lentinula edodes. Fungal Genet. Biol. 118, 37-44. doi: 10.1016/j.fgb.2018.07.002

Wang, L., Wu, X., Gao, W., Zhao, M., Zhang, J., and Huang, C. (2017). Differential expression patterns of Pleurotus ostreatus catalase genes during developmental stages and under heat stress. Genes 8, 335. doi: 10.3390/genes8110335

Xie, J. L., Bohovych, I., Wong, E. O., Lambert, J. P., Gingras, A. C., Khalimonchuk, O., et al. (2017). Ydj1 governs fungal morphogenesis and stress response, and facilitates mitochondrial protein import via Mas1 and Mas2. Microbial Cell 4, 342-361. doi: 10.15698/mic2017.10.594

Yang, G., Tang, L., Gong, Y., Xie, J., Fu, Y., Jiang, D., et al. (2018). A cerato-platanin protein SsCP1 targets plant PR1 and contributes to virulence of Sclerotinia sclerotiorum. New Phytol. 217, 739-755. doi: 10.1111/nph.14842

Yin, C., Zheng, L., Zhu, J., Chen, L., and Ma, A. (2015). Enhancing stress tolerance by overexpression of a methionine sulfoxide reductase A (MsrA) gene in Pleurotus ostreatus. Appl. Microbiol. Biot. 99, 3115-3126. doi: 10.1007/s00253014-6365-4

Zhong, X., Yang, J., Shi, Y., Wang, X., and Wang, G. L. (2018). The DnaJ protein OsDjA6 negatively regulates rice innate immunity to the blast fungus Magnaporthe oryzae. Mol. Plant Pathol. 19, 607-614. doi: 10.1111/mpp.12546

Zhou, S. S., Wang, G. Z., Luo, Y., Ma, C. J., Gong, Y. H., Bian, Y. B., et al. (2018). Auxin and auxin analogues enhancing the thermotolerance of Lentinula edodes. Mycosystema 37, 1723-1730.

Zhou, W., Zhou, T., Li, M. X., Zhao, C. L., Jia, N., Wang, X. X., et al. (2012). The Arabidopsis J-protein AtDjB1 facilitates thermotolerance by protecting cells against heat-induced oxidative damage. New Phytol. 194, 364-378. doi: 10.1111/j.1469-8137.2012.04070.x

Zhu, X., Liang, S., Yin, J., Yuan, C., Wang, J., Li, W., et al. (2015). The DnaJ OsDjA7/8 is essential for chloroplast development in rice (Oryza sativa). Gene 574, 11-19. doi: 10.1016/j.gene.2015.07.067

Conflict of Interest: The authors declare that the research was conducted in the absence of any commercial or financial relationships that could be construed as a potential conflict of interest.

Copyright (c) 2020 Wang, Luo, Wang, Zhou, Mou, Kang, Xiao, Bian and Gong. This is an open-access article distributed under the terms of the Creative Commons Attribution License (CC BY). The use, distribution or reproduction in other forums is permitted, provided the original author(s) and the copyright owner(s) are credited and that the original publication in this journal is cited, in accordance with accepted academic practice. No use, distribution or reproduction is permitted which does not comply with these terms. 